
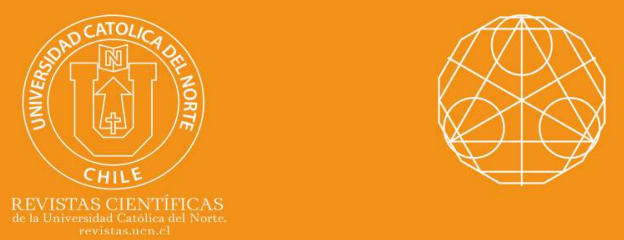

\title{
Another example of the mutual singularity of multifractal measures
}

Zied Douzi ${ }^{1}$ (1) orcid.org/0000-0003-3108-8511

Amal Samti ${ }^{2}$ (10 orcid.org/0000-0001-8621-1009

Bilel Selmi ${ }^{3}$ (1) orcid.org/0000-0001-8823-6699

University of Monastir, Dept. of Mathematics, Analysis, Probability and Fractals Laboratory LR18ES17. Monastir, Tunisia.

1酒zied.douzi@fsm.rnu.tn ; ${ }^{2}$ amal_samti@yahoo.fr; ${ }^{3}$ bilel.selmi@fsm.rnu.tn

\section{Abstract:}

We propose an example for which the multifractal Hausdorff and packing measures are mutually singular.

Keywords: Multifractal analysis; Homogeneous Moran fractals; Homogeneous Moran Measures.

MSC (2020): 28A20, 28A78, 28A80.

\section{Cite this article as (IEEE citation style):}

Zied Douzi, Amal Samti, and Bilel Selmi, "Another example of the mutual singularity of multifractal measures", Proyecciones (Antofagasta, On line), vol. 40, no. 1, pp. 17-33, 2021, doi: 10.22199/issn.0717-6279-2021-01-0002

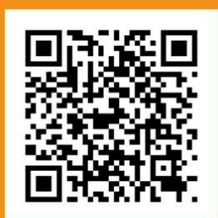

Article copyright: (C) 2021 Zied Douzi, Amal Samti and Bilel Selmi. This is an open access article distributed under the terms of the Creative Commons License, which permits unrestricted use and distribution provided the original author and source are credited. 


\section{Introduction}

Let $\mu$ be a probability measure on a metric space $X$. The Hausdorff multifractal spectrum function, $f_{\mu}$, and the packing multifractal spectrum function, $F_{\mu}$, of the measure $\mu$ are defined respectively by

$$
f_{\mu}(\alpha)=\operatorname{dim}_{H}(E(\alpha)) \quad \text { and } \quad F_{\mu}(\alpha)=\operatorname{dim}_{P}(E(\alpha)) \text { for } \alpha \geq 0,
$$

where

$$
E(\alpha)=\left\{x \in \operatorname{supp} \mu ; \lim _{r \rightarrow 0} \frac{\log (\mu(B(x, r)))}{\log r}=\alpha\right\},
$$

and $\operatorname{supp} \mu$ is the topological support of $\mu$.

During the past 25 years there has been an enormous interest in computing the multifractal spectra of measures in the mathematical literature. Particularly, the multifractal spectra of various classes of measures in Euclidean space $\mathbf{R}^{n}$ exhibiting some degree of self-similarity have been computed rigorously. The reader can be referred to the paper [9], the textbooks $[7,11]$ and the references therein. Some heuristic arguments using tech- niques of statistical mechanics (see [8]) show that the singularity spectrum should be finite on a compact interval, noted by $\operatorname{Dom}(\mu)$, and is expected to be the Legendre transform conjugate of the $\tau_{\mu}$-function, given by

$$
\tau_{\mu}(q)=\lim _{r \rightarrow 0} \frac{1}{-\log r} \log \left(\sup \left\{\sum_{i} \mu\left(B\left(x_{i}, r\right)\right)^{q}\right\}\right)
$$

where the supremum is taken over all centered packing $\left(B\left(x_{i}, r\right)\right)_{i}$ of $\operatorname{supp} \mu$. That is, for all $\alpha \in \operatorname{Dom}(\mu)$,

$$
f_{\mu}(\alpha)=\inf _{q \in \mathbf{R}}\left\{\alpha q+\tau_{\mu}(q)\right\}=: \tau_{\mu}^{*}(\alpha)
$$

The multifractal formalism (1.1) has been proved rigorously for random and non-random self-similar measures, for self-conformal measures, for self-affine measures and for Moran measures. We notice that the proofs of the multifractal formalism (1.1) in the above-mentioned references (see for example $[3,9,13]$ and references therein) are all based on the same key idea. The upper bound for $f_{\mu}(\alpha)$ is obtained by a standard covering argu- ment, involving Besicovitch's covering theorem or Vitali's covering theorem. 
However, its lower bound is usually much harder to prove and is related to the existence of an auxiliary measure (Gibbs measures) which is supported by the set to be analyzed. In an attempt to develop a general theoretical framework for studying the multifractal structure of arbitrary measures, Olsen [9], Pesin [11] and Peyrière [12] suggested various ways of defining measures analogous to those of Gibbs measures in very general settings. For an arbitrary Borel probability measure $\mu$ on $\mathbf{R}^{n}$, they introduced two parameter families of measures,

$$
\left\{H_{\mu}^{q, t} ; q, t \in \mathbf{R}\right\} \quad \text { and } \quad\left\{P_{\mu}^{q, t} ; q, t \in \mathbf{R}\right\}
$$

based on certain generalizations of the Hausdorff measure and of the packing measure. For $q, t \in \mathbf{R}, E \subseteq \mathbf{R}^{n}$ and $\delta>0$, write

$$
\bar{P}_{\mu, \delta}^{q, t}(E)=\sup \left\{\sum_{i} \mu\left(B\left(x_{i}, r_{i}\right)\right)^{q}\left(2 r_{i}\right)^{t}\right\}, \quad E=\emptyset,
$$

where the supremum is taken over all centered $\delta$-packing of $E$. Moreover we can set $\bar{P}_{\mu, \delta}^{q, t}(\emptyset)=0$. Also, we define

$$
\bar{H}_{\mu, \delta}^{q, t}(E)=\inf \left\{\sum_{i} \mu\left(B\left(x_{i}, r_{i}\right)\right)^{q}\left(2 r_{i}\right)^{t}\right\}, \quad E=\emptyset,
$$

where the infinimum is taken over all centered $\delta$-covering of $E$. Moreover we can set $\bar{H}_{\mu, \delta}^{q, t}(\emptyset)=0$. Especially, we have the conventions $0^{q}=\infty$ for $q \leq 0$ and $0^{q}=0$ for $q>0$.

The packing and Hausdorff pre-measures are defined respectively by

$$
\bar{P}_{\mu}^{q, t}(E)=\inf _{\delta>0} \bar{P}_{\mu, \delta}^{q, t}(E) \text { and } \bar{H}_{\mu}^{q, t}(E)=\sup _{\delta>0} \bar{H}_{\mu, \delta}^{q, t}(E) .
$$

The function $\bar{P}_{\mu}^{q, t}$ is not necessarily countably subadditive, also the set function $\bar{H}_{\mu}^{q, t}$ is not necessarily monotone. For these reasons, Olsen introduced the packing and Hausdorff measures denoted respectively by $P_{\mu}^{q, t}$ and $H_{\mu}^{q, t}$ are defined as following

$$
P_{\mu}^{q, t}(E)=\inf _{E \subseteq \bigcup_{i} E_{i}} \sum_{i} \bar{P}_{\mu}^{q, t}\left(E_{i}\right) \quad \text { and } \quad H_{\mu}^{q, t}(E)=\sup _{F \subseteq E} \bar{H}_{\mu}^{q, t}(F) .
$$

The functions $H_{\mu}^{q, t}$ and $P_{\mu}^{q, t}$ are metric outer measures and thus measures on the Borel family of subsets of $\mathbf{R}^{n}$. It is easy to see that $P_{\mu}^{q, t} \leq \bar{P}_{\mu}^{q, t}$. 
Moreover, by using Besicovitch's theorem, there exists an integer $\xi \in \mathbf{N}$, such that $H_{\mu}^{q, t} \leq \xi P_{\mu}^{q, t}$ (see [9]). The measure $H_{\mu}^{q, t}$ is a multifractal generalization of the centered Hausdorff measure, whereas $P_{\mu}^{q, t}$ is a multifractal generalization of the packing measure. In fact, in the case when $t \geq 0$, $H_{\mu}^{0, t}=H^{t}$ and $P_{\mu}^{0, t}=P^{t}$, where $H^{t}$ denotes the t-dimensional centered Hausdorff measure and $P^{t}$ denotes the t-dimensional packing measure.

The measures $H_{\mu}^{q, t}$ and $P_{\mu}^{q, t}$ and the pre-measure $\bar{P}_{\mu}^{q, t}$ assign in the usual way a multifractal dimension to each subset $E$ of $\mathbf{R}^{n}$. They are respectively denoted by $b_{\mu}^{q}(E), B_{\mu}^{q}(E)$ and $\Lambda_{\mu}^{q}(E)$ and satisfy

$$
\begin{gathered}
b_{\mu}^{q}(E)=\inf \left\{t \in \mathbf{R} ; \quad H_{\mu}^{q, t}(E)=0\right\}, \quad B_{\mu}^{q}(E)=\inf \left\{t \in \mathbf{R} ; \quad P_{\mu}^{q, t}(E)=0\right\}, \\
\Lambda_{\mu}^{q}(E)=\inf \left\{t \in \mathbf{R} ; \quad \bar{P}_{\mu}^{q, t}(E)=0\right\} .
\end{gathered}
$$

The number $b_{\mu}^{q}(E)$ is an obvious multifractal analogue of the Hausdorff dimension $\operatorname{dim}_{H}(E)$ of $E$ whereas $B_{\mu}^{q}(E)$ and $\Lambda_{\mu}^{q}(E)$ are obvious multi-

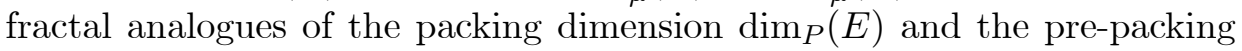
dimension $\Delta(E)$ of $E$ respectively. In fact, it follows immediately from the definitions that

$$
\operatorname{dim}_{H}(E)=b_{\mu}^{0}(E), \quad \operatorname{dim}_{P}(E)=B_{\mu}^{0}(E) \quad \text { and } \quad \Delta(E)=\Lambda_{\mu}^{0}(E) .
$$

Next, for $q \in \mathbf{R}$, we define the dimension functions $b_{\mu}, B_{\mu}$ and $\Lambda_{\mu}$ by

$$
b_{\mu}(q)=b_{\mu}^{q}(\operatorname{supp} \mu), \quad B_{\mu}(q)=B_{\mu}^{q}(\operatorname{supp} \mu) \text { and } \Lambda_{\mu}(q)=\Lambda_{\mu}^{q}(\operatorname{supp} \mu) .
$$

It is well known that the functions $b_{\mu}, B_{\mu}$ and $\Lambda_{\mu}$ are decreasing and $B_{\mu}, \Lambda_{\mu}$ are convex and satisfying $b_{\mu} \leq B_{\mu} \leq \Lambda_{\mu}$.

One of the main importance of the multifractal measures $H_{\mu}^{q, t}$ and $P_{\mu}^{q, t}$, and the corresponding dimension functions $b_{\mu}, B_{\mu}$, and $\Lambda_{\mu}$ is due to the fact that the multifractal spectra functions $f_{\mu}$ and $F_{\mu}$ are bounded above by the Legendre transforms of $b_{\mu}$ and $B_{\mu}$, respectively, i.e.,

$$
\operatorname{dim}_{H}(E(\alpha)) \leq b_{\mu}^{*}(\alpha) \quad \text { and } \quad \operatorname{dim}_{P}(E(\alpha)) \leq B_{\mu}^{*}(\alpha) \text { for all } \quad \alpha \geq 0 .
$$

These inequalities may be viewed as rigorous versions of the multifractal formalism. Furthermore, for many natural families of measures we have

$$
\operatorname{dim}_{H}(E(\alpha))=b_{\mu}^{*}(\alpha) \quad \text { and } \quad \operatorname{dim}_{P}(E(\alpha))=B_{\mu}^{*}(\alpha) \quad \text { for some } \quad \alpha \geq 0,
$$


see for example $[3,9,10,13]$. It is clear by comparing the definitions of the measures $H_{\mu}^{q, t}$ and $P_{\mu}^{q, t}$, and definition of the $\tau_{\mu}$-function which appears in the multifractal formalism that $b_{\mu}(q)$ and $B_{\mu}(q)$ are mathematically rigor- ous versions of $\tau_{\mu}(q)$, and that the one-parameter families

$$
\left\{H_{\mu}^{q, b_{\mu}(q)} ; q \in \mathbf{R}\right\} \quad \text { and } \quad\left\{P_{\mu}^{q, B_{\mu}(q)} ; q \in \mathbf{R}\right\}
$$

play the role of the auxiliary measures $\left\{\mu_{q} ; q \in \mathbf{R}\right\}$. In particular, we would expect that the measures $\left\{H_{\mu}^{q, b_{\mu}(q)} ; q \in \mathbf{R}\right\}$ and $\left\{P_{\mu}^{q, B_{\mu}(q)} ; q \in \mathbf{R}\right\}$ have similar properties to those of the auxiliary measures $\left\{\mu_{q} ; q \in \mathbf{R}\right\}$. This has been proved rigorously for self-similar, quasi self-similar, self-conformal measures and for arbitrary measures, see for example $[3,9,10]$ and references therein.

Let us mention that the interest of mathematicians in singularly continuous measures and probability distributions were fairly weak, which can be explained, on the one hand, by the absence of adequate analytic apparatus for specification and investigation of these measures, and, on the other hand, by a widespread opinion about the absence of applications of these measures. Due to the fractal explosion and a deep connection between the theory of fractals and singular measures, the situation has radically changed in the last years. The multifractal and the fractal analysis allows one to perform a certain classification of these measures. Therefore, Olsen in [9], posed the following two questions:

Question 1: Let $p, q \in \mathbf{R}$ and assume that $b_{\mu}$ is differentiable at $p$ and $q$ with $b_{\mu}^{\prime}(p) \neq b_{\mu}^{\prime}(q)$. Then, the following problem remains open:

$$
H_{\mu}^{p, b_{\mu}(p)}{ }_{\lfloor\text {supp } \mu} \perp H_{\mu}^{q, b_{\mu}(q)}{ }_{\lfloor\text {supp } \mu} .
$$

Question 2: Let $p, q \in \mathbf{R}$ and assume that $B_{\mu}$ is differentiable at $p$ and $q$ with $B_{\mu}^{\prime}(p) \neq B_{\mu}^{\prime}(q)$. Then, the following problem remains open:

$$
P_{\mu}^{p, B_{\mu}(p)}{ }_{\lfloor\text {supp } \mu} \perp P_{\mu}^{q, B_{\mu}(q)}{ }_{\lfloor\text {supp } \mu} .
$$

In $[6,9]$, the authors discussed these questions and provided some examples of the mutual singularity of multifractal Hausdorff and packing measures for graph directed self-similar measures in $\mathbf{R}^{n}$ with totally disconnected support, cookie-cutter measures [9], for some homogeneous Moran measures [6] and for self-similar measures satisfying the significantly weaker open set condition $[4,5]$. In this paper, we give another example for which the multifractal Hausdorff and packing measures are mutually singular. 


\section{Main result}

\subsection{Moran set}

Let recall the class of homogeneous Moran sets. One consider $\left\{n_{k}\right\}_{k \geq 1}$ a sequence of positive integers and $\left\{\psi_{k}\right\}_{k \geq 1}$ a sequence of positive real vectors with

$$
\psi_{k}=\left(b_{k 1}, b_{k 2}, \ldots, b_{k n_{k}}\right), \quad \sum_{j=1}^{n_{k}} b_{k j} \leq 1, \quad k \in \mathbf{N} .
$$

Let $\mathcal{D}_{0}=\emptyset$, and for any $k \geq 1$, set

$\mathcal{D}_{m, k}=\left\{\left(i_{m}, i_{m+1}, \ldots, i_{k}\right) ; \quad 1 \leq i_{j} \leq n_{j}, \quad m \leq j \leq k\right\} \quad$ and $\quad \mathcal{D}_{k}=\mathcal{D}_{1, k}$.

We also define $\mathcal{D}=\bigcup_{k \geq 0} \mathcal{D}_{k}$. If $\sigma=\left(\sigma_{1}, \ldots, \sigma_{k}\right) \in \mathcal{D}_{k}, \theta=\left(\theta_{1}, \ldots, \theta_{m}\right) \in$ $\mathcal{D}_{k+1, m}$, we denote $\sigma * \theta=\left(\sigma_{1}, \ldots, \sigma_{k}, \theta_{1}, \ldots, \theta_{m}\right)$.

Definition 2.1. Let $I$ be a closed interval such that $|I|=1$. We say the collection $F=\left\{I_{\sigma}, \sigma \in D\right\}$ of closed subsets of I fulfills the Moran structure if it satisfies the following conditions (MSC):

(a) $I_{\emptyset}=I$

(b) For all $k \geq 0$ and $\sigma \in \mathcal{D}_{k}, I_{\sigma * 1}, I_{\sigma * 2}, \ldots, I_{\sigma * n_{k+1}}$ are subintervals of $I_{\sigma}$, and satisfy

$$
I_{\sigma * i}^{\circ} \cap I_{\sigma * j}^{\circ}=\emptyset, \quad \text { whenever } i \neq j\left(A^{\circ} \text { denotes the interior of } A .\right)
$$

(c) For any $k \geq 1, \sigma \in \mathcal{D}_{k-1}, \quad b_{k}=\frac{\left|I_{\sigma * j}\right|}{\left|I_{\sigma}\right|}, \quad 1 \leq j \leq n_{k}$ where $|A|$ denotes the diameter of $A$.

Let $F$ be a collection of closed subintervals of $I$ having homogeneous Moran structure. The set $E(F)=\bigcap_{k \geq 0} \bigcup_{\sigma \in \mathcal{D}_{k}} I_{\sigma}$ is called an homogeneous Moran set determined by $F$.

Let $F_{k}=\left\{I_{\sigma}, \sigma \in \mathcal{D}_{k}\right\}$, and $F=\bigcup_{k \geq 0} F_{k}$. The elements of $F_{k}$ are called the basic elements of order $k$ of the Moran set $E$ and the elements of $F$ are called the basic elements of the Moran set $E$.

Remark 2.1. If $\lim _{n \rightarrow+\infty} \sup _{\sigma \in \mathcal{D}_{n}}\left|I_{\sigma}\right|>0$, then E contains interior points. Thus the measure and dimension properties will be trivial. We assume therefore $\lim _{n \rightarrow+\infty} \sup _{\sigma \in \mathcal{D}_{n}}\left|I_{\sigma}\right|=0$. 
Suppose that the set $I$, the sequences $\left\{n_{k}\right\}$ and $\left\{\psi_{k}\right\}$ are given, we denote by $\mathcal{M}=: \mathcal{M}\left(I,\left\{n_{k}\right\},\left\{\psi_{k}\right\}\right)$ the class of the Moran sets satisfying the (MSC) and call the Moran class associated with the triplet $\left(I,\left\{n_{k}\right\},\left\{\psi_{k}\right\}\right)$.

Now let $\mathcal{O}=\left\{a_{1}, a_{2}, \ldots, a_{m}\right\}$ and for $\omega \in \mathcal{O}^{\mathbf{N}}:=\left\{s_{1} s_{2} \cdots s_{k} \cdots, s_{i} \in \mathcal{O}\right\}$, write

$$
\omega_{k}=\left.\omega\right|_{k}=s_{1} s_{2} \cdots s_{k}, \text { then }\left|\omega_{k}\right|=k .
$$

We also denote by $\left|\omega_{k}\right|_{a_{i}}$ the number of occurrences of the letter $a_{i}$ in $\omega_{k}$. Given a probability vector $\gamma=\left(\gamma_{1}, \gamma_{2}, \ldots, \gamma_{m}\right)$, we say $\omega$ has frequency vector $\gamma$, if $\lim _{k \rightarrow+\infty} \frac{\left|\omega_{k}\right|_{a_{i}}}{k}=\gamma_{i}>0$, for every $a_{i} \in \mathcal{O}$. It is easy see that

$$
\sum_{i=1}^{m}\left|\omega_{k}\right|_{a_{i}}=k \quad \text { and } \quad \sum_{j=1}^{m} \gamma_{j}=1
$$

For $\gamma=\left(\gamma_{1}, \gamma_{2}, \ldots, \gamma_{m}\right)$, we consider the set

$$
\mathcal{O}_{\gamma}^{\mathbf{N}}=\left\{\omega=\left\{s_{k}\right\}_{k \geq 1} ; \quad s_{k} \in \mathcal{O}, \lim _{k \rightarrow+\infty} \frac{\left|\omega_{k}\right|_{a_{i}}}{k}=\gamma_{i}, 1 \leq i \leq m\right\} .
$$

From now on, suppose $m_{i} \in \mathbf{N}$, for $1 \leq i \leq m$ and $\psi_{i}=\left(b_{i 1}, b_{i 2}, \ldots, b_{i m_{i}}\right)$ be a positive real vector with $\sum_{j=1}^{m_{i}} b_{i j} \leq 1$. For $\omega \in \mathcal{O}_{\gamma}^{\mathbf{N}}$, in the Moran construction above, for any $k \geq 1$ if $s_{k}=a_{i}$ take $n_{k}=m_{i}$. Then we construct the Moran set relting to $\omega \in \mathcal{O}_{\gamma}^{\mathbf{N}}$ and we denote it by $E(\omega)=\left\{I,\left\{n_{k}\right\},\left\{\psi_{k}\right\}\right\}$. From [13], we have

$$
\operatorname{dim}_{H} E(\omega)=\liminf _{k \rightarrow+\infty} d_{k} \quad \text { and } \quad \operatorname{dim}_{P} E(\omega)=\limsup _{k \rightarrow+\infty} d_{k}
$$

where $d_{k}$ satisfies the equation

$$
\prod_{i=1}^{k} \sum_{j=1}^{n_{i}} b_{i j}^{d_{k}}=1
$$

It follows from (2.1) that

$$
\prod_{i=1}^{m}\left(\sum_{j=1}^{n_{i}} b_{i j}^{d_{k}}\right)^{\left|\omega_{k}\right|_{a_{i}}}=1
$$


Now taking the logarithms, the lower and upper limits respectively as $k$ tends to infinity, we get

$$
\operatorname{dim}_{H} E(\omega)=\operatorname{dim}_{P} E(\omega)=d
$$

where the number $d$ satisfies the following equation

$$
\sum_{i=1}^{m} \gamma_{i} \log \left(\sum_{j=1}^{m_{i}} b_{i j}^{d}\right)=0
$$

Suppose $I_{\sigma} \in \mathcal{F}_{k}, k \geq 1$, and let $I_{\sigma * 1}, I_{\sigma * 2}, \ldots, I_{\sigma * n_{k+1}}$ be the $n_{k+1}$ basic intervals of order $k+1$ contained in $I_{\sigma}$ arranged from the left to the right. For all $1 \leq j \leq n_{k+1}-1$, let $\operatorname{dist}\left(I_{\sigma * j}, I_{\sigma *(j+1)}\right) \geq \Delta_{k}\left|I_{\sigma}\right|$, where $\left\{\Delta_{k}\right\}$ is a sequence of positive reals. We set $\Delta=\inf _{k>1} \Delta_{k}$.

\subsection{Moran measure}

Now we will define a Borel probability measure on the Moran set $E(\omega)$.

Given $P_{a_{i}}=\left(p_{i 1} p_{i 2} \cdots p_{i m_{i}}\right), 1 \leq i \leq m$ be probability vectors, i.e.,

$$
p_{i j}>0 \text { and } \sum_{j=1}^{m_{i}} p_{i j}=1, \text { for } 1 \leq i \leq m .
$$

Next, for $k \geq 1, \sigma \in \mathcal{D}_{k}$, we know that $\sigma=\sigma_{1} \sigma_{2} \cdots \sigma_{k} \in \mathcal{D}_{k}$ where $\sigma_{k} \in\left\{1,2, \ldots, m_{i}\right\}$, if $s_{k}=a_{i}$. For $\sigma=\sigma_{1} \sigma_{2} \cdots \sigma_{k}$, consider $\sigma\left(a_{i}\right)$ as follows: let $\omega_{k}=s_{1} \cdots s_{k}, e_{1}<e_{2}<\cdots e_{\left|\omega_{k}\right|_{a_{i}}}$ be the occurrences of the letter $a_{i}$ in $\omega_{k}$, then $\sigma\left(a_{i}\right)=\sigma_{e_{1}} \sigma_{e_{2}} \cdots \sigma_{e_{\left|\omega_{k}\right|_{a_{i}}}}$. By convention, we write $\sigma\left(a_{i}\right)=\sigma_{i_{1}} \sigma_{i_{2}} \cdots \sigma_{i\left|\omega_{k}\right|_{a_{i}}}$, where $\sigma_{i_{j}} \in\left\{1,2, \ldots, m_{i}\right\}$ for $1 \leq i \leq m$. In fact, $\sigma_{\left(a_{1}\right)} * \sigma_{\left(a_{2}\right)} * \cdots * \sigma_{\left(a_{m}\right)}$ is a rearrangement of $\sigma=\sigma_{1} \cdots \sigma_{k}$. We make the convention that $\sigma\left(a_{i}\right)=\emptyset$ if $\left|\omega_{k}\right|_{a_{i}}=0$. From now on, we consider

$$
p_{\sigma\left(a_{i}\right)}=p_{i \sigma_{i 1}} \cdots p_{i \sigma_{i}\left|\omega_{k}\right|_{a_{i}}}, \quad 1 \leq i \leq m .
$$

It is easy to see that $\sum_{\sigma \in \mathcal{D}_{k}} \prod_{i=1}^{m} p_{\sigma\left(a_{i}\right)}=1$ for every $k \geq 1$. We make the convention that

$$
p_{\sigma\left(a_{i}\right)}=1 \text { if } \sigma\left(a_{i}\right)=\emptyset .
$$


Let $\mu$ be a mass distribution on $E(\omega)$, such that for any $I_{\sigma} \in \mathcal{F}_{k}, \sigma \in \mathcal{D}_{k}$

$$
\mu\left(I_{\sigma}\right)=p_{\sigma\left(a_{1}\right)} p_{\sigma\left(a_{2}\right)} \cdots p_{\sigma\left(a_{m}\right)} \quad \text { and } \quad \mu\left(\sum_{\sigma \in \mathcal{D}_{k}} I_{\sigma}\right)=1 .
$$

Since $\mu$ is related with $\omega$, we denote it by $\mu(\omega)$. In this case $\mu(\omega)$ is called Moran measure on $E(\omega)$. By the construction of the set $E(\omega)$, we write $b_{\sigma\left(a_{i}\right)}=b_{i \sigma_{i 1}} \cdots b_{i \sigma_{i}\left|\omega_{k}\right|_{a_{i}}}$ for $1 \leq i \leq m$, then $\left|I_{\sigma}\right|=\prod_{i=1}^{m} b_{\sigma\left(a_{i}\right)}$ and $\# \mathcal{F}_{k}=\prod_{i=1}^{m} m_{i}^{\left|\omega_{k}\right|_{a_{i}}}$. In the next, for simplicity we denote $E(\omega)$ by $E$, and $\mu(\omega)$ by $\mu$.

Now one consider an auxiliary function $\beta$ as follows: for every $q \in \mathbf{R}$ and $k \geq 1$, there is a unique number $\beta_{k}(q)$ such that

$$
\sum_{\sigma \in \mathcal{D}_{k}} p_{\sigma}^{q}\left|I_{\sigma}\right|^{\beta_{k}(q)}=1
$$

Thus, we prove by a simple calculation that

$$
\prod_{i=1}^{m}\left(\sum_{j=1}^{m_{i}} p_{i j}^{q} b_{i j}^{\beta_{k}(q)}\right)^{\left|\omega_{k}\right|_{a_{i}}}=1 .
$$

Now taking the logarithms, the lower and upper limits respectively when $k$ tends to infinity, we obtain

$$
\liminf _{k \rightarrow+\infty} \beta_{k}(q)=\limsup _{k \rightarrow+\infty} \beta_{k}(q)=\beta(q)
$$

where $\beta(q)$ satisfies the equation

$$
\sum_{i=1}^{m} \gamma_{i} \log \left(\sum_{j=1}^{m_{i}} p_{i j}^{q} b_{i j}^{\beta(q)}\right)=0 .
$$

The following result describes some properties of the function $\beta$.

Proposition 2.1. [13] For all $q \in \mathbf{R}$, the function $\beta$ satisfies the following statements

1. $\beta(0)=\lim _{k \rightarrow+\infty} d_{k}=d, \beta(1)=0$. 
2. $\beta(q)$ is strictly decreasing, and $\lim _{q \rightarrow \mp \infty} \beta(q)= \pm \infty$.

3. $\beta(q)$ is convex in $q$, and is strictly convex if and only if the quantity $\frac{\log p_{i j}}{\log b_{i j}}$ is not the same for all $1 \leq j \leq m_{i}, i=1,2, \ldots, m$.

Our main result is the following.

Theorem 2.1. Suppose that $\Delta>0$. Then, for all $p, q \in \mathbf{R}$ with $\beta^{\prime}(p) \neq$ $\beta^{\prime}(q)$ we have

$$
H_{\mu}^{p, \beta(p)} \perp H_{\mu}^{q, \beta(q)} \quad \text { and } \quad P_{\mu}^{p, \beta(p)} \perp P_{\mu}^{q, \beta(q)} \quad \text { on } \quad E .
$$

\section{Proof of the main result}

Let $\mu$ be a Borel probability measure on $\mathbf{R}^{n}$ and $a>1$, we write

$$
D_{a}(\mu)=\limsup _{r \searrow 0}\left(\sup _{x \in \operatorname{supp} \mu} \frac{\mu(B(x, a r))}{\mu(B(x, r))}\right) .
$$

We will now say that the measure $\mu$ satisfies the doubling condition if there exists $a>1$ such that $D_{a}(\mu)<\infty$. It is easily seen that the exact value of the parameter $a$ is unimportant: $D_{a}(\mu)<\infty$, for some $a>1$ if and only if $D_{a}(\mu)<\infty$, for all $a>1$. In particular, if $\mu$ satisfies the doubling condition then $H_{\mu}^{q, t} \leq P_{\mu}^{q, t}$.

Given $q \in \mathbf{R}$, it follows from [13, Proposition 3.1] that there exists a probability measure $\nu_{q}$ supported by $E$ such that for any $k \geq$ 1 and $\sigma_{0} \in \mathcal{D}_{k}$,

$$
\nu_{q}\left(I_{\sigma_{0}}\right)=\frac{\mu\left(I_{\sigma_{0}}\right)^{q}\left|I_{\sigma_{0}}\right|^{\beta(q)}}{\sum_{\sigma \in \mathcal{D}_{k}} \mu\left(I_{\sigma}\right)^{q}\left|I_{\sigma}\right|^{\beta(q)}} .
$$

However, in [13] it is shown that

$$
\lim _{r \downarrow 0} \frac{\log \mu(B(x, r))}{\log r}=-\beta^{\prime}(q), \quad \nu_{q}-\mathrm{a} . \mathrm{s}
$$

which implies that $\nu_{q}\left(E\left(-\beta^{\prime}(q)\right)\right)=1$. We therefore infer that if $p, q \in \mathbf{R}$ with $\beta^{\prime}(p) \neq \beta^{\prime}(q)$, then 


$$
\nu_{p} \perp \nu_{q}
$$

Next, we present some tools, as well as lemmas, which will be used in the proof of our main result.

Lemma 3.1. We have

$$
0<\liminf _{k \rightarrow+\infty} \sum_{\sigma \in \mathcal{D}_{k}} \mu\left(I_{\sigma}\right)^{q}\left|I_{\sigma}\right|^{\beta(q)} \leq \limsup _{k \rightarrow+\infty} \sum_{\sigma \in \mathcal{D}_{k}} \mu\left(I_{\sigma}\right)^{q}\left|I_{\sigma}\right|^{\beta(q)}<+\infty
$$

Proof. By a simple calculation, we can get $\beta(q)-\beta_{k}(q)=O\left(\frac{1}{k}\right)$. By using (2.1), we get

$$
\begin{gathered}
\sum_{\sigma \in \mathcal{D}_{k}} \mu\left(I_{\sigma}\right)^{q}\left|I_{\sigma}\right|^{\beta(q)}=\left|I_{\sigma}\right|^{\beta(q)-\beta_{k}(q)} \geq \\
\left(\min \left\{b_{i j} ; 1 \leq j \leq m_{i}, 1 \leq i \leq m\right\}\right)^{k\left(\beta(q)-\beta_{k}(q)\right)},
\end{gathered}
$$

which implies that

$$
\liminf _{k \rightarrow+\infty} \sum_{\sigma \in \mathcal{D}_{k}} \mu\left(I_{\sigma}\right)^{q}\left|I_{\sigma}\right|^{\beta(q)}>0
$$

The proof of the

$$
\limsup _{k \rightarrow+\infty} \sum_{\sigma \in \mathcal{D}_{k}} \mu\left(I_{\sigma}\right)^{q}\left|I_{\sigma}\right|^{\beta(q)}<+\infty .
$$

is identical to the proof of the statement in the first part and is therefore omitted.

Lemma 3.2. There exists a constant $\underline{K}>0$ such that for any $q \in \mathbf{R}$

$$
\underline{K} \nu_{q}(E) \leq H_{\mu}^{q, \beta(q)}(E)
$$

Proof. For convenience of presentation let $I_{n}(x)$ be the $n$ th-level basic set of $E$ containing the point $x$. Fix $\delta>0$ and let $\left(B\left(x_{n}, r_{n}\right)\right)_{n \in \mathbf{N}}$ be a centered $\delta$-covering of $E$. For each $n$ we can choose $\sigma(n) \in \mathcal{D}_{k}$, for any $k \geq 1$ such 
that $x_{n} \in I_{\sigma(n)}$. Since $\Delta>0$, then for each $n \in \mathbf{N}$ we can find $h_{n}, \ell_{n} \in \mathbf{N}$ such that

$$
\left|I_{\sigma(n) \mid h_{n}+1}\right| \leq r_{n}<\left|I_{\sigma(n) \mid h_{n}}\right| \quad \text { and } \quad \Delta\left|I_{\sigma(n) \mid \ell_{n}+1}\right| \leq r_{n}<\Delta\left|I_{\sigma(n) \mid \ell_{n}}\right|
$$

which implies that

$(3.2) I_{\sigma(n) \mid h_{n}+1}\left(x_{n}\right) \subseteq B\left(x_{n}, r_{n}\right) \quad$ and $\quad E \cap B\left(x_{n}, r_{n}\right) \subseteq I_{\sigma(n) \mid \ell_{n}+1}\left(x_{n}\right)$.

So,

$$
\begin{aligned}
\nu_{q}(E) & \leq \sum_{n} \nu_{q}\left(B\left(x_{n}, r_{n}\right)\right) \\
& \leq \sum_{n} \nu_{q}\left(I_{\sigma(n) \mid \ell_{n}+1}\left(x_{n}\right)\right) \\
& =\sum_{n} \frac{\mu\left(I_{\sigma(n) \mid \ell_{n}+1}\left(x_{n}\right)\right)^{q}\left|I_{\sigma(n) \mid \ell_{n}+1}\right|^{\beta(q)}}{\sum_{\sigma \in \mathcal{D}_{\ell_{n}+1}} \mu\left(I_{\sigma}\right)^{q}\left|I_{\sigma}\right|^{\beta(q)}} \\
& \leq k_{1} \sum_{n} \mu\left(I_{\sigma(n) \mid \ell_{n}+1}\left(x_{n}\right)\right)^{q}\left|I_{\sigma(n) \mid \ell_{n}+1}\right|^{\beta(q)} .
\end{aligned}
$$

If $\beta(q) \geq 0$, then

$$
\left|I_{\sigma(n) \mid \ell_{n}+1}\right|^{\beta(q)} \leq(2 \Delta)^{\beta(q)}\left(2 r_{n}\right)^{\beta(q)} .
$$

Moreover, if $\beta(q)<0$, one has for $s_{\ell_{n}+1}=a_{i}$

$$
\left|I_{\sigma(n) \mid \ell_{n}+1}\right|=b_{i j_{0}}\left|I_{\sigma(n) \mid \ell_{n}}\right|, \quad j=1,2, \ldots, m_{i},
$$

since

$$
\left|I_{\sigma(n) \mid \ell_{n}+1}\right| \geq \min \left\{b_{i j} ; 1 \leq j \leq m_{i}, 1 \leq i \leq m\right\} \cdot\left|I_{\sigma(n) \mid l_{n}}\right|
$$

then

$$
2 r_{n} \leq 2 \Delta\left|I_{\sigma(n) \mid \ell_{n}}\right| \leq \frac{2 \Delta}{\min \left\{b_{i j} ; 1 \leq j \leq m_{i}, 1 \leq i \leq m\right\}}\left|I_{\sigma(n) \mid \ell_{n}+1}\right| .
$$

Which implies that

$$
\left|I_{\sigma(n) \mid \ell_{n}+1}\right|^{\beta(q)} \leq\left(\frac{\min \left\{b_{i j} ; 1 \leq j \leq m_{i}, 1 \leq i \leq m\right\}}{2 \Delta}\right)^{\beta(q)}\left(2 r_{n}\right)^{\beta(q)} .
$$


Thus we proved the following inequality

$$
\left|I_{\sigma(n) \mid \ell_{n}+1}\right|^{\beta(q)} \leq c_{1}\left(2 r_{n}\right)^{\beta(q)}
$$

where $c_{1}$ is a suitable constant. If $q<0$, using (3.2), we obtain

$$
\mu\left(I_{\sigma(n) \mid \ell_{n}+1}\left(x_{n}\right)\right)^{q} \leq \mu\left(B\left(x_{n}, r_{n}\right)\right)^{q} .
$$

For the case $q \geq 0$, since the measure $\mu$ satisfies the doubling condition (see [14, Proposition 3.2]), there exists a constant $A>0$ such that

$\mu\left(I_{\sigma(n) \mid \ell_{n}+1}\left(x_{n}\right)\right)^{q} \leq\left(\frac{\mu\left(B\left(x_{n}, \frac{r_{n}}{\Delta}\right)\right)}{\mu\left(B\left(x_{n}, r_{n}\right)\right)}\right)^{q} \mu\left(B\left(x_{n}, r_{n}\right)\right)^{q} \leq A^{q} \mu\left(B\left(x_{n}, r_{n}\right)\right)^{q}$.

From (3.5) and (3.6), there exists a constant $c_{2}$ such that

$$
\mu\left(I_{\sigma(n) \mid \ell_{n}+1}\left(x_{n}\right)\right)^{q} \leq c_{2} \mu\left(B\left(x_{n}, r_{n}\right)\right)^{q} .
$$

Now combining (3.3), (3.4) and (3.7), we obtain

$$
\nu_{q}(E) \leq k_{1} c_{1} c_{2} \sum_{n} \mu\left(B\left(x_{n}, r_{n}\right)\right)^{q}\left(2 r_{n}\right)^{\beta(q)} .
$$

Consequently

$$
\underline{K} \nu_{q}(E) \leq \bar{H}_{\mu, \delta}^{q, \beta(q)}(E) \leq \bar{H}_{\mu}^{q, \beta(q)}(E) \leq H_{\mu}^{q, \beta(q)}(E)
$$

where $\underline{K}=\left(k_{1} c_{1} c_{2}\right)^{-1}$, which achieves the proof of Lemma 3.2.

Lemma 3.3. There exists a constant $\bar{K}>0$ such that for any $q \in \mathbf{R}$

$$
P_{\mu}^{q, \beta(q)}(E) \leq \bar{K} \nu_{q}(E) .
$$

Proof. Let $F$ be a closed subset of $E$. For $\delta>0$ write

$$
B(F, \delta)=\{x \in E ; \quad \operatorname{dist}(x, F) \leq \delta\} .
$$

Since $F$ is closed, $B(F, \delta) \searrow F$ for $\delta \searrow 0$. Then for all $\varepsilon>0$, there exists $\delta_{0}$ satisfying

$$
\nu_{q}(B(F, \delta)) \leq \nu_{q}(F)+\varepsilon, \quad \forall 0<\delta<\delta_{0} .
$$


Fix $\delta>0$ and let $\left(B\left(x_{n}, r_{n}\right)\right)_{n}$ be a centered $\delta$-packing of $F$. For each integer $n$, choose $\sigma(n) \in \mathcal{D}_{k}$, for any $k \geq 1$ such that $x_{n} \in I_{\sigma(n)}$ and pick $h_{n}, \ell_{n} \in \mathbf{N}$ such that

$$
\left|I_{\sigma(n) \mid h_{n}+1}\right| \leq r_{n}<\left|I_{\sigma(n) \mid h_{n}}\right| \text { and } \quad \Delta\left|I_{\sigma(n) \mid \ell_{n}+1}\right| \leq r_{n}<\Delta\left|I_{\sigma(n) \mid \ell_{n}}\right| .
$$

Observing that

$$
I_{\sigma(n) \mid h_{n}+1}\left(x_{n}\right) \subseteq B\left(x_{n}, r_{n}\right) \quad \text { and } \quad E \cap B\left(x_{n}, r_{n}\right) \subseteq I_{\sigma(n) \mid \ell_{n}+1}(x) .
$$

By using the same technics as that in Lemma 3.2 , there exists $\widetilde{c}_{1}, \widetilde{c}_{2}>0$ such that

$$
\left(2 r_{n}\right)^{\beta(q)} \leq \widetilde{c}_{1}\left|I_{\sigma(n) \mid h_{n}+1}\right|^{\beta(q)} \quad \text { and } \quad \mu\left(B\left(x_{n}, r_{n}\right)\right)^{q} \leq \widetilde{c}_{2} \mu\left(I_{\sigma(n) \mid h_{n}+1}\left(x_{n}\right)\right)^{q} .
$$

Thus,

$$
\begin{aligned}
\sum_{n} \mu\left(B\left(x_{n}, r_{n}\right)\right)^{q}\left(2 r_{n}\right)^{\beta(q)} \leq & \widetilde{c}_{1} \widetilde{c}_{2} \sum_{n} \mu\left(I_{\sigma(n) \mid h_{n}+1}\left(x_{n}\right)\right)^{q}\left|I_{\sigma(n) \mid h_{n}+1}\right|^{\beta(q)} \\
\leq & \widetilde{c}_{1} \widetilde{c}_{2} \sum_{n}\left(\frac{\mu\left(I_{\sigma(n) \mid h_{n}+1}\left(x_{n}\right)\right)^{q}\left|I_{\sigma(n) \mid h_{n}+1}\right|^{\beta(q)}}{\sum_{\sigma \in \mathcal{D}_{h_{n}+1}} \mu\left(I_{\sigma}\right)^{q}\left|I_{\sigma}\right|^{\beta(q)}}\right) \\
& \times \sum_{\sigma \in \mathcal{D}_{h_{n}+1}} \mu\left(I_{\sigma}\right)^{q}\left|I_{\sigma}\right|^{\beta(q)} \\
\leq & \widetilde{k}_{1} \widetilde{c}_{1} \widetilde{c}_{2} \sum_{n} \nu_{q}\left(I_{\sigma(n) \mid h_{n}+1}\left(x_{n}\right)\right) \\
\leq & \bar{K} \sum_{n} \nu_{q}\left(B\left(x_{n}, r_{n}\right)\right) \\
\leq & \bar{K} \nu_{q}(B(F, \delta)) \leq \bar{K}\left(\nu_{q}(F)+\varepsilon\right) \\
\leq & \bar{K}\left(\nu_{q}(E)+\varepsilon\right) \text { where } \bar{K}=\widetilde{k}_{1} \widetilde{c}_{1} \widetilde{c}_{2} .
\end{aligned}
$$

It results that

$$
\bar{P}_{\mu}^{q, \beta(q)}(F) \leq \bar{K}\left(\nu_{q}(E)+\varepsilon\right) .
$$


Letting $\varepsilon \downarrow 0$, now yields

$$
P_{\mu}^{q, \beta(q)}(E) \leq \bar{P}_{\mu}^{q, \beta(q)}(E) \leq \bar{K} \nu_{q}(E) .
$$

Which complete the proof of Lemma 3.3.

Let us now prove our main theorem.

Proof of Theorem 2.1. By using Lemma 3.2, Lemma 3.3 and since $\mu$ satisfies the doubling condition, one has

$$
\underline{K} \nu_{q} \leq H_{\mu}^{q, \beta(q)} \leq P_{\mu}^{q, \beta(q)} \leq \bar{K} \nu_{q} \quad \text { on } \quad E .
$$

It results that

$$
\frac{1}{\bar{K}} H_{\mu}^{q, \beta(q)} \leq \nu_{q} \leq \frac{1}{\underline{K}} H_{\mu}^{q, \beta(q)} \quad \text { on } \quad E
$$

and

$$
\frac{1}{\bar{K}} P_{\mu}^{q, \beta(q)} \leq \nu_{q} \leq \frac{1}{\underline{K}} P_{\mu}^{q, \beta(q)} \quad \text { on } \quad E .
$$

Finally, the result follows from (3.1).

Remark 3.1. The results of Theorem 2.1 hold if we replace the multifractal Hausdorff and packing measures by the multifractal Hewitt-Stromberg measures (see $[1,2]$ for the precise definitions), which in particular provides an answer to [2, Questions 4 and 5].

Remark 3.2. It follows from Lemma 3.2 and Lemma 3.3 that

$$
b_{\mu}(q)=B_{\mu}(q)=\Lambda_{\mu}(q)=\beta(q), \quad \forall q \in \mathbf{R} .
$$

It is also instructive to consider the special case $q=0$. In particular, we have

$$
\operatorname{dim}_{H}(E)=\operatorname{dim}_{P}(E)=\Delta(E)=\beta(0)=d .
$$




\section{References}

[1] N. Attia and B. Selmi, "Regularities of multifractal Hewitt-Stromberg measures", Communication Korean Mathematical Society, vol.34, no. 1, pp. 213-230, Jan. 2019, doi: 10.4134/ CKMS.c180030

[2] N. Attia and B. Selmi, "A multifractal formalism for Hewitt-Stromberg measures", Journal of geometric analysis, Oct., 2019, doi: 10.1007/s12220-019-00302-3

[3] F. Ben Nasr, I. Bhouri and Y. Heurteaux, "The validity of the multifractal formalism: results and examples", Advances in mathematics, vol. 165, no. 2, pp. 264-284, Feb. 2002, doi: 10.1006/ aima.2001.2025

[4] M. Das, "Pointwise Local Dimensions", Ph.D. Thesis, The Ohio State University, USA, 1996.

[5] M. Das, "Hausdorff measures, dimensions and mutual singularity", Transactions of the American Mathematical Society, vol. 357, no. 11, pp. 4249-4268, Nov. 2005, doi: 10.1090/ S0002-9947-05-04031-6

[6] Z. Douzi and B. Selmi, "On the mutual singularity of multifractal measures”, Electronical research archives, vol. 28, no. 1, pp. 423-432, Mar. 2020, doi: 10.3934/ era.2020024

[7] K. J. Falconer, Techniques in fractal geometry, New York, NY: Wiley, 1997.

[8] T. C. Halsey, M. H. Jensen, L. P. Kadanof, I. Procaccia, and B. J. Shraiman, "Fractal measures and their singularities: the characterization of strange sets", Physics Revision A., vol. 33, pp. 1141-1151, Feb. 1986, doi: 10.1103/ PhysRevA.33.1141

[9] L. Olsen, "A multifractal formalism", Advances in mathematics, vol. 116, no.1, pp. 82-196, Nov. 1995, doi: 10.1006/ aima.1995.1066

[10] L. Olsen, "Dimension inequalities of multifractal Hausdorff measures and multifractal packing measures", Mathematica scandinavica, vol. 86, no.1, pp. 109-129, 2000, doi: 10.7146/ math.scand.a-14284

[11] Y. Pesin, Dimension theory in dynamical systems, Contemporary views and applications, Chicago, IL: University of Chicago Press, 1997. 
[12] J. Peyrière, "Multifractal measures," in Probabilistic and stochastic methods in analysis, with applications, J. S. Byrnes, J. L. Byrnes, K. A. Hargreaves, and K. Berry, Eds. Dordrecht: Springer, 1992, pp. 175-186.

[13] M. Wu, "The multifractal spectrum of some Moran measures", Science in China Series A: Mathematics volume, vol. 48, pp. 1097-1112, Aug. 2005, doi: 10.1360/ 022004-10 